\title{
Freud's superpotential in general relativity and in Einstein-Cartan theory
}

\author{
Christian G. Böhmer ${ }^{1, *}$ and Friedrich W. Hehl ${ }^{2, \dagger}$ \\ ${ }^{1}$ Department of Mathematics, University College London, \\ Gower Street, London, WCIE 6BT, United Kingdom \\ ${ }^{2}$ Institute for Theoretical Physics, University of Cologne, Zülpicher Strasse 77, 50937 Köln, Germany and \\ Department of Physics and Astronomy, University of Missouri Columbia, Missouri 65201, USA
}

(Received 15 December 2017; published 21 February 2018)

\begin{abstract}
The identification of a suitable gravitational energy in theories of gravity has a long history, and it is well known that a unique answer cannot be given. In the first part of this paper we present a streamlined version of the derivation of Freud's superpotential in general relativity. It is found if we once integrate the gravitational field equation by parts. This allows us to extend these results directly to the Einstein-Cartan theory. Interestingly, Freud's original expression, first stated in 1939, remains valid even when considering gravitational theories in Riemann-Cartan or, more generally, in metric-affine spacetimes.
\end{abstract}

DOI: $10.1103 /$ PhysRevD.97.044028

\section{INTRODUCTION}

\section{A. Gravity and energy}

General Relativity (GR) does not lend itself naturally to the definition of gravitational energy. This led to the formulation of energy via pseudotensors or an energymomentum density complex; see Chen, Nester, and Tung [1] for a brief historical account; earlier contributions, chronologically ordered, include [2-10]. These objects appear more or less naturally when studying gravitational field equations, depending on one's approach. In the present paper we proceed by revisiting the Einstein field equation written in exterior calculus. This has the great advantage that a single integration by parts allows us to define a conserved quantity, which can be related directly with Freud's superpotential [11]. Within the context of general relativity, most of these results are known and can be found in the literature.

Our approach allows us to extend these results smoothly to other gravitational theories. First, it will be applied to Einstein-Cartan theory where we will derive the analogue superpotential in the presence of torsion. Second, we show how the formalism naturally includes all metric-affine gravity theories. It is particularly noteworthy that Freud's original "affine tensor density" applies to all such theories despite Freud's proof being given only in the case of general relativity.

\section{B. Notation and conventions}

For exterior calculus and its application to field theory, we recommend, as examples, Bamberg and Sternberg [12],

\footnotetext{
c.boehmer@ucl.ac.uk

hehl@thp.uni-koeln.de
}

Agricola and Friedrich [13], and Scheck [14]. We take our corresponding conventions from [15] (see also [16]). For tensor calculus, see Schouten $[17,18]$ and, for the generalized Kronecker symbols and the $\epsilon$-system, in particular Sokolnikoff [19].

The coframe one-form is denoted by $\vartheta^{\alpha}$, with Greek (anholonomic) indices $\alpha, \beta, \gamma, \ldots=0,1,2,3$. The vector frame $e_{\beta}$ is dual to the coframe, that is, the interior product of the frame as applied to the coframe reads $\left.e_{\beta}\right\rfloor \vartheta^{\alpha}=\delta_{\beta}^{\alpha}$. (Holonomic) coordinate indices are written in Latin, namely $i, j, k, \ldots=0,1,2,3$. A $p$-form $\Psi$ expanded with respect to the anholonomic basis is given by

$$
\Psi=\frac{1}{p !} \Psi_{\alpha_{1} \ldots \alpha_{p}} \vartheta^{\alpha_{1}} \wedge \cdots \wedge \vartheta^{\alpha_{p}} .
$$

The $\eta$-basis is defined in the conventional way; see [15]. If we take the interior product $\rfloor$ of an arbitrary frame $e_{\alpha}$ with the metric volume element 4-form $\eta:=$ $\sqrt{\left|\operatorname{det} g_{\mu \nu}\right|} \vartheta^{0} \wedge \vartheta^{1} \wedge \vartheta^{2} \wedge \vartheta^{3}$, then we find a 3-form $\eta_{\alpha}$; if we contract again, we find a 2 -form $\eta_{\alpha \beta}$, etc.:

$$
\begin{gathered}
\left.\eta_{\alpha}:=e_{\alpha}\right\rfloor \eta=\frac{1}{3 !} \eta_{\alpha \beta \gamma \delta} \vartheta^{\beta} \wedge \vartheta^{\gamma} \wedge \vartheta^{\delta}={ }^{\star} \vartheta_{\alpha}, \\
\left.\eta_{\alpha \beta}:=e_{\beta}\right\rfloor \eta_{\alpha}=\frac{1}{2 !} \eta_{\alpha \beta \gamma \delta} \vartheta^{\gamma} \wedge \vartheta^{\delta}={ }^{\star}\left(\vartheta_{\alpha} \wedge \vartheta_{\beta}\right), \\
\left.\eta_{\alpha \beta \gamma}:=e_{\gamma}\right\rfloor \eta_{\alpha \beta}=\frac{1}{1 !} \eta_{\alpha \beta \gamma \delta} \vartheta^{\delta}={ }^{\star}\left(\vartheta_{\alpha} \wedge \vartheta_{\beta} \wedge \vartheta_{\gamma}\right), \\
\left.\left.\left.\left.\left.\eta_{\alpha \beta \gamma \delta}:=e_{\delta}\right\rfloor \eta_{\alpha \beta \gamma}=e_{\delta}\right\rfloor e_{\gamma}\right\rfloor e_{\beta}\right\rfloor e_{\alpha}\right\rfloor \eta \\
={ }^{\star}\left(\vartheta_{\alpha} \wedge \vartheta_{\beta} \wedge \vartheta_{\gamma} \wedge \vartheta_{\delta}\right) .
\end{gathered}
$$

Similar formulas are valid for the $\epsilon$-system, where $\epsilon_{\alpha \beta \gamma \delta}=$ $\pm 1,0$ is the Levi-Citita symbol; see $[15,20]$. The Hodge 
star ${ }^{\star}$ is then substituted by the diamond star ${ }^{\diamond}$. This system will be used in metric-affine spacetimes.

In Riemannian geometry a tilde above an expression $\tilde{M}$ is used. This means such quantities are based on the Christoffel symbol components without torsion or nonmetricity. In Riemann-Cartan space the corresponding quantities are denoted without a tilde. The torsion 2-form and the curvature 2-forms read, respectively, as

$$
\begin{aligned}
T^{\alpha} & :=D \vartheta^{\alpha}=d \vartheta^{a}+\Gamma_{\beta}{ }^{\alpha} \wedge \vartheta^{\beta}, \\
R_{\alpha}{ }^{\beta} & =d \Gamma_{\alpha}{ }^{\beta}-\Gamma_{\alpha}{ }^{\gamma} \wedge \Gamma_{\gamma}{ }^{\beta} .
\end{aligned}
$$

Einstein's gravitational constant and the speed of light are put to one: $\kappa=1, c=1$.

\section{FREUD'S SUPERPOTENTIAL IN GENERAL RELATIVITY}

\section{A. General relativity in exterior calculus}

It is possible to express Einstein's field equation [21] in the framework of the calculus of exterior differential forms; see Misner, Thorne, and Wheeler [22], Trautman [23], Thirring and Wallner [10,24], and Kopczyński [25]. Let $\tilde{R}_{\alpha}{ }^{\beta}$ be the curvature 2-form of the (pseudo)Riemannian spacetime,

$$
\tilde{R}_{\alpha}^{\beta}=d \tilde{\Gamma}_{\alpha}^{\beta}-\tilde{\Gamma}_{\alpha}^{\gamma} \wedge \tilde{\Gamma}_{\gamma}^{\beta}
$$

with $\tilde{\Gamma}_{\alpha}{ }^{\beta}$ as the Levi-Civita connection 1-form. We call the Einstein 3-form $\tilde{G}_{\alpha}$. Then the Einstein equation reads [23]

$$
\tilde{G}_{\alpha}:=\frac{1}{2} \eta_{\alpha \beta \gamma} \wedge \tilde{R}^{\beta \gamma}=\mathcal{T}_{\alpha}
$$

Here the source term $\mathcal{T}_{\alpha}$ represents the symmetric Hilbert energy-momentum 3 -form of matter. The usual symmetric energy-momentum tensor of rank 2 is given by

$$
T_{\alpha \beta}={ }^{\star}\left(\vartheta_{\beta} \wedge \mathcal{T}_{\alpha}\right) .
$$

To recover the standard form of the Einstein field equations, where the Einstein tensor has the form of a trace-reversed Ricci tensor, one can isolate the components of the Einstein 3-form using (1) and (4). Finally, one can apply the Hodge dual to obtain the 1-form whose components will be the usual Einstein tensor components.

If one desires to introduce a superpotential $a ̀$ la Freud [11], then one has to isolate in (8) a leading term of the general form $\sim d\left(\eta_{. .} \wedge \tilde{\Gamma}\right)$. In exterior calculus, it is straightforward to see how to achieve this. We substitute (7) into (8):

$$
\frac{1}{2} \eta_{\alpha \beta \gamma} \wedge\left(d \tilde{\Gamma}^{\beta \gamma}-\tilde{\Gamma}^{\beta \delta} \wedge \tilde{\Gamma}_{\delta}^{\gamma}\right)=\mathcal{T}_{\alpha}
$$

The next step is now particularly simple; we use the Leibniz rule and find immediately

$$
\begin{aligned}
& d\left\{-\frac{1}{2} \eta_{\alpha \beta \gamma} \wedge \tilde{\Gamma}^{\beta \gamma}\right\}+\frac{1}{2}\left(d \eta_{\alpha \beta \gamma}\right) \wedge \tilde{\Gamma}^{\beta \gamma}-\frac{1}{2} \eta_{\alpha \beta \gamma} \wedge \tilde{\Gamma}^{\beta \delta} \wedge \tilde{\Gamma}_{\delta}^{\gamma} \\
& =\mathcal{T}_{\alpha} .
\end{aligned}
$$

It is possible to manipulate the second term on the left-hand side of this equation: We recall that in a Riemannian space $\tilde{D} \eta_{\alpha \beta \gamma}=0$. Thus,

$$
\begin{aligned}
0 & =d \eta_{\alpha \beta \gamma}-\tilde{\Gamma}_{\alpha}{ }^{\delta} \wedge \eta_{\delta \beta \gamma}-\tilde{\Gamma}_{\beta}{ }^{\delta} \wedge \eta_{\alpha \delta \gamma}-\tilde{\Gamma}_{\gamma}{ }^{\delta} \wedge \eta_{\alpha \beta \delta} \\
& =d \eta_{\alpha \beta \gamma}-3 \tilde{\Gamma}_{[\alpha}{ }^{\delta} \wedge \eta_{\beta \gamma] \delta},
\end{aligned}
$$

which implies

$$
d \eta_{\alpha \beta \gamma}=3 \tilde{\Gamma}_{[\alpha}^{\delta} \wedge \eta_{\beta \gamma] \delta}
$$

We substitute (13) into (11) and find, after some algebra,

$$
d\left\{-\frac{1}{2} \eta_{\alpha \beta \gamma} \wedge \tilde{\Gamma}^{\beta \gamma}\right\}-\eta_{\beta \gamma[\alpha} \wedge \tilde{\Gamma}_{\delta]}^{\beta} \wedge \tilde{\Gamma}^{\delta \gamma}=\mathcal{T}_{\alpha} .
$$

One is now led to define the Freud 2-form $[11]^{1}$

$$
\tilde{\mathcal{F}}_{\alpha}:=-\frac{1}{2} \eta_{\alpha \beta \gamma} \wedge \tilde{\Gamma}^{\beta \gamma}
$$

and the gravitational energy-momentum, the TrautmanSparling 3-form [27],

$$
\tilde{t}_{\alpha}:=\eta_{\beta \gamma[\alpha} \wedge \tilde{\Gamma}_{\delta]}^{\beta} \wedge \tilde{\Gamma}^{\delta \gamma} .
$$

Then we can rewrite (14) simply as

$$
d \tilde{\mathcal{F}}_{\alpha}=\tilde{t}_{\alpha}+\mathcal{T}_{\alpha}
$$

Equation (17) has been derived earlier also by Frauendiener [3], for example. However, he finds it "somewhat startling to see the Einstein tensor to show up in this connection" [8c]. In our deduction, the relation to the Einstein 3-form is apparent and quite natural. A similar observation can be found in [1] where it was pointed out that this derivation of the superpotential is in "remarkable constrast" to the tensor calculus approach.

\footnotetext{
${ }^{1}$ According to Kopczyński [26], Trautman, around 1975, taught about gravitational radiation at Warsaw University. There Kopczyński learned from him about the forms $\tilde{\mathcal{F}}_{\alpha}$ and $\tilde{t}_{\alpha}$. Nurowski remembers that Kopczyński lectured about these forms in the early 1980s. Later Trautman spoke about them in Erice and, probably, from Erice it went somehow to Vienna. These forms then appeared in papers of Thirring. Incidentally, Trautman called $\tilde{F}_{\alpha}$ Freud's superpotential.
} 
Our main result is respresented by Eq. (17), together with definitions (15) and (16). Clearly, because of Poincaré's lemma, $d d \tilde{\mathcal{F}}_{\alpha}=0$. Accordingly, we find the conservation law

$$
d\left(\tilde{t}_{\alpha}+\mathcal{T}_{\alpha}\right)=0
$$

This demonstrates that the nontensorial 3 -form $\tilde{t}_{\alpha}$ represents an energy complex of the gravitational field; see also Schrödinger [28].

It turns out that the Freud 2-form $\tilde{\mathcal{F}}_{\alpha}$ is not exactly the object used by Freud in the derivation of the energy complex but rather its Hodge dual. This is addressed in the following.

\section{B. Hodge dual of the Freud two-form}

In subsequent parts we will need the Hodge dual of the Freud two-form. By applying the standard formulas for the Hodge star, we find

$$
\begin{aligned}
{ }^{\star} \mathcal{F}_{\alpha} & ={ }^{\star}\left(-\frac{1}{2} \eta_{\alpha \beta \gamma} \wedge \tilde{\Gamma}^{\beta \gamma}\right)=-\frac{1}{2}{ }^{\star}\left(\eta_{\alpha \beta \gamma \delta} \vartheta^{\delta} \wedge \tilde{\Gamma}^{\beta \gamma}\right) \\
& =-\frac{1}{2} \eta_{\alpha \beta \gamma \delta}{ }^{\star}\left(\vartheta^{\delta} \wedge \tilde{\Gamma}^{\beta \gamma}\right)=-\frac{1}{2} \eta_{\alpha \beta \gamma \delta}{ }^{\star}\left(\vartheta^{\delta} \wedge \vartheta^{\varepsilon} \tilde{\Gamma}_{\varepsilon}^{\beta \gamma}\right) \\
& =-\frac{1}{2} \eta_{\alpha \beta \gamma \delta} \tilde{\Gamma}_{\varepsilon}^{\beta \gamma \star}\left(\vartheta^{\delta} \wedge \vartheta^{\varepsilon}\right) .
\end{aligned}
$$

Accordingly, we find the relatively compact formula $\left(\eta^{\delta \zeta}=-\eta^{\zeta \delta}\right)$

$$
{ }^{\star} \mathcal{F}_{\alpha}=\frac{1}{2} \eta_{\alpha \beta \gamma \delta} g^{\beta \varepsilon} \tilde{\Gamma}_{\zeta \varepsilon}^{\gamma} \eta^{\zeta \delta}
$$

The components of this expression are given implicitly by ${ }^{\star} \mathcal{F}_{\alpha}=\frac{1}{2}{ }^{\star} \mathcal{F}_{\mu \nu \alpha} \quad \vartheta^{\mu} \wedge \vartheta^{\nu}$ or explicitly by ${ }^{\star} \mathcal{F}_{\mu \nu \alpha}=$ $\left.\left.e_{\nu}\right\rfloor e_{\mu}\right\rfloor^{\star} \mathcal{F}_{\alpha}$. Thus, Eq. (20) yields

$$
\begin{aligned}
{ }^{\star} \mathcal{F}_{\mu \nu \alpha} & \left.\left.=e_{\nu}\right\rfloor e_{\mu}\right\rfloor\left(\frac{1}{2} \eta_{\alpha \beta \gamma \delta} g^{\beta \varepsilon} \tilde{\Gamma}_{\zeta \varepsilon}^{\gamma} \eta^{\zeta \delta}\right) \\
& \left.\left.=\frac{1}{2} \eta_{\alpha \beta \gamma \delta} g^{\beta \varepsilon} \tilde{\Gamma}_{\zeta \varepsilon}^{\gamma} e_{\nu}\right\rfloor e_{\mu}\right\rfloor \eta^{\zeta \delta} \\
& =\frac{1}{2} \eta_{\alpha \beta \gamma \delta} g^{\beta \varepsilon} \tilde{\Gamma}_{\zeta \varepsilon}^{\gamma} \eta^{\zeta \delta}{ }_{\mu \nu}=\frac{1}{2}\left(\eta_{\alpha \beta \gamma \delta} \eta_{\mu \nu}{ }^{\zeta \delta}\right) g^{\beta \varepsilon} \tilde{\Gamma}_{\zeta \varepsilon}^{\gamma} .
\end{aligned}
$$

It appears that the last expression becomes more transparent if we raise the indices $\mu$ and $\nu$ :

$$
{ }^{\star} \mathcal{F}^{\mu \nu}{ }_{\alpha}=\frac{1}{2}\left(\eta_{\alpha \beta \gamma \delta} \eta^{\mu \nu \zeta \delta}\right) g^{\beta \varepsilon} \tilde{\Gamma}_{\zeta \varepsilon}{ }^{\gamma}=\frac{1}{2} \delta_{\alpha \beta \gamma}^{\mu \nu \zeta} g^{\beta \varepsilon} \tilde{\Gamma}_{\zeta \varepsilon}{ }^{\gamma} .
$$

In the last transformation, we used the rules for the $\eta$-system; see [[19] Eq. (40.5)] together with the generalized Kronecker deltas. ${ }^{2}$ The object $\delta_{\alpha \beta \gamma}^{\mu \nu \zeta}$ can be written as a determinant:

\footnotetext{
${ }^{2}$ The generalized Kroneckers and the related $\epsilon$-system are also discussed in [[20] Ch.A.1].
}

$$
\delta_{\alpha \beta \gamma}^{\mu \nu \zeta}=\left|\begin{array}{ccc}
\delta_{\alpha}^{\mu} & \delta_{\alpha}^{\nu} & \delta_{\alpha}^{\zeta} \\
\delta_{\beta}^{\mu} & \delta_{\beta}^{\nu} & \delta_{\beta}^{\zeta} \\
\delta_{\gamma}^{\mu} & \delta_{\gamma}^{\nu} & \delta_{\gamma}^{\zeta}
\end{array}\right| .
$$

If we substitute this into (22), we find eventually

$$
{ }^{\star} \mathcal{F}^{\mu \nu}{ }_{\alpha}=\frac{1}{2}\left|\begin{array}{ccc}
\delta_{\alpha}^{\mu} & \delta_{\alpha}^{\nu} & \delta_{\alpha}^{\zeta} \\
g^{\mu \varepsilon} & g^{\nu \varepsilon} & g^{\zeta \varepsilon} \\
\tilde{\Gamma}_{\zeta \varepsilon}{ }^{\mu} & \tilde{\Gamma}_{\zeta \varepsilon}{ }^{\nu} & \tilde{\Gamma}_{\zeta \varepsilon}{ }^{\zeta}
\end{array}\right| .
$$

This result is analogous to the one found by Freud in holonomic coordinates, as we will see.

Using the notation $\mathfrak{g}^{i k}:=\sqrt{|g|} g^{i k}$ Freud wrote the expression of the superpotential in a compact form by using the determinant

$$
\mathfrak{A}^{i n}{ }_{k}=\frac{1}{2}\left|\begin{array}{ccc}
\delta_{k}^{i} & \delta_{k}^{n} & \delta_{k}^{m} \\
\mathfrak{g}^{i r} & \mathfrak{g}^{n r} & \mathfrak{g}^{m r} \\
\tilde{\Gamma}_{m r}{ }^{i} & \tilde{\Gamma}_{m r}{ }^{n} & \tilde{\Gamma}_{m r}{ }^{m}
\end{array}\right|=-\mathfrak{A}^{n i}{ }_{k} .
$$

Clearly, ${ }^{\star} \mathcal{F}^{\mu \nu}{ }_{\alpha}$ and $\mathfrak{A}^{i n}{ }_{k} / \sqrt{|g|}$ are the same mathematical object. The former is expressed in arbitrary (anholonomic) frames, whereas the latter is expressed in terms of curvilinear (holonomic) coordinates.

\section{EINSTEIN-CARTAN AND METRIC-AFFINE GRAVITY}

\section{A. Freud in Einstein-Cartan theory (EC)}

The field equations of EC, see, e.g., Trautman [23,29], Blagojević and Hehl [30], or Obukhov [31,32], are algebraic in $R^{\alpha \beta}$ and $T^{\alpha}$, respectively. In spite of this, we want to try to put them in a form that is reminiscent of the field equations of Yang-Mills type:

$\underbrace{G_{\alpha}}_{\text {Einstein 3-form }}:=\frac{1}{2} \eta_{\alpha \beta \gamma} \wedge R^{\beta \gamma}=\mathfrak{I}_{\alpha} \Rightarrow d \mathcal{F}_{\alpha}-t_{\alpha}=\mathfrak{I}_{\alpha}$,

$\underbrace{P_{\alpha \beta}}_{\text {Palatini 3-form }}:=\frac{1}{2} \eta_{\alpha \beta \gamma} \wedge T^{\gamma}=\mathfrak{S}_{\alpha \beta} \Rightarrow d(\cdots)-s_{\alpha \beta}=\mathfrak{S}_{\alpha \beta}$.

The sources on the right-hand sides of the two field equations are the canonical 3-forms of energy-momentum and spin angular momentum of matter, respectively.

Our deduction of a Freud 2-form will proceed in strict analogy to the one in general relativity. We only have to drop the tildes. There is, however, one difference: In a RiemannCartan space, the covariant exterior derivatives of the $\eta$-forms do not vanish any longer. We rather have (see [15]) 


$$
\begin{aligned}
D \eta_{\alpha} & =T^{\delta} \wedge \eta_{\alpha \delta}, \\
D \eta_{\alpha \beta} & =T^{\delta} \wedge \eta_{\alpha \beta \delta}, \\
D \eta_{\alpha \beta \gamma} & =T^{\delta} \wedge \eta_{\alpha \beta \gamma \delta}, \\
D \eta_{\alpha \beta \gamma \delta} & =0 .
\end{aligned}
$$

As a consequence of (30), we now find, instead of the Riemannian result (13), the corresponding relation in a Riemann-Cartan space as

$$
d \eta_{\alpha \beta \gamma}=3 \Gamma_{[\alpha}^{\delta} \wedge \eta_{\beta \gamma] \delta}+T^{\delta} \wedge \eta_{\alpha \beta \gamma \delta} .
$$

This newly emerging torsion term is the basic difference between both deductions. Interestingly, the torsion term will not enter the Freud superpotential explicitly.

Analogous to that above, we substitute the gravitational field strengths curvature $R^{\beta \gamma}$ and the torsion $T^{\gamma}$ into the two field equations:

$$
\begin{gathered}
\frac{1}{2} \eta_{\alpha \beta \gamma} \wedge\left(d \Gamma^{\beta \gamma}-\Gamma^{\beta \delta} \wedge \Gamma_{\delta}^{\gamma}\right)=\mathfrak{T}_{\alpha}, \\
\frac{1}{2} D \eta_{\alpha \beta}=d\left(\frac{1}{2} \eta_{\alpha \beta}\right)+\Gamma_{[\alpha}^{\gamma} \wedge \eta_{\beta] \gamma}=\mathfrak{S}_{\alpha \beta} .
\end{gathered}
$$

We partially integrate the first term of (33) and find immediately

$$
\begin{aligned}
& d(\underbrace{-\frac{1}{2} \eta_{\alpha \beta \gamma} \wedge \Gamma^{\beta \gamma}}_{\mathcal{F}_{\alpha}})+\frac{1}{2}\left(d \eta_{\alpha \beta \gamma}\right) \wedge \Gamma^{\beta \gamma}-\frac{1}{2} \eta_{\alpha \beta \gamma} \wedge \Gamma^{\beta \delta} \wedge \Gamma_{\delta}^{\gamma} \\
& =\mathfrak{T}_{\alpha} .
\end{aligned}
$$

Now we substitute (32) into (35) and collect the terms containing the $\Gamma$ 's quadratically:

$$
\begin{aligned}
& d\left(-\frac{1}{2} \eta_{\alpha \beta \gamma} \wedge \Gamma^{\beta \gamma}\right)-\eta_{\beta \gamma[\alpha} \wedge \Gamma_{\delta]}^{\beta} \wedge \Gamma^{\delta \gamma}+\frac{1}{2} \eta_{\alpha \beta \gamma \delta} \Gamma^{\beta \gamma} \wedge T^{\delta} \\
& =\mathfrak{T}_{\alpha} .
\end{aligned}
$$

Following the GR calculation closely, we define the first term as the Freud 2-form

$$
\mathcal{F}_{\alpha}:=-\frac{1}{2} \eta_{\alpha \beta \gamma} \wedge \Gamma^{\beta \gamma}
$$

and, enriched by a torsion term, the gravitational energymomentum 3-form

$$
t_{\alpha}:=\eta_{\beta \gamma[\alpha} \wedge \Gamma_{\delta]}^{\beta} \wedge \Gamma^{\delta \gamma}-\frac{1}{2} \eta_{\alpha \beta \gamma \delta} \Gamma^{\beta \gamma} \wedge T^{\delta} .
$$

Thus, the first field equation of EC can simply be written as

$$
d \mathcal{F}_{\alpha}-t_{\alpha}=\mathfrak{T}_{\alpha} .
$$

The second field equation is already in its final form. However, for compactness we introduce the gravitational spin 3-form

$$
s_{\alpha \beta}:=-\Gamma_{[\alpha}^{\gamma} \wedge \eta_{\beta] \gamma} .
$$

Then the second field equation of EC reads as follows:

$$
d\left(\frac{1}{2} \eta_{\alpha \beta}\right)-s_{\alpha \beta}=\mathfrak{S}_{\alpha \beta} .
$$

If we define the energy-momentum and spin complexes

$$
\check{\mathfrak{I}}_{\alpha}:=t_{\alpha}+\mathfrak{\mathfrak { T }}_{\alpha}, \quad \check{\mathfrak{S}}_{\alpha \beta}:=s_{\alpha \beta}+\mathfrak{\mathfrak { S }}_{\alpha \beta},
$$

the field equations and the energy-momentum and spin laws look even simpler:

$$
\begin{aligned}
d \mathcal{F}_{\alpha} & =\check{\mathfrak{I}}_{\alpha}, \quad d\left(\frac{1}{2} \eta_{\alpha \beta}\right)=\check{\mathfrak{S}}_{\alpha \beta} \quad \text { with } \quad d \check{\mathfrak{I}}_{\alpha}=0, \\
d \check{\mathfrak{S}}_{\alpha \beta} & =0 .
\end{aligned}
$$

The conservation equations are again implied by the Poincaré lemma. This was previously studied by Obukhov et al. [33,34]; see also Shimizu [35].

\section{B. Hodge dual of the Freud two-form in Einstein-Cartan theory}

As we saw in the definition of the Freud 2-form in a Riemann-Cartan space in (37), the formula looks exactly like its Riemannian equivalent; only the tilde got lost. Accordingly, the computation of the Hodge dual in Einstein-Cartan exactly parallels the one in a Rieamannian space. We find again the compact formula

$$
{ }^{\star} \mathcal{F}_{\alpha}=\frac{1}{2} \eta_{\alpha \beta \gamma \delta} g^{\beta \varepsilon} \Gamma_{\zeta \varepsilon} \eta^{\zeta \delta} ;
$$

the tilde is now missing. In turn, the components of (44) read

$$
{ }^{\star} \mathcal{F}^{\mu \nu}{ }_{\alpha}=\frac{1}{2}\left(\eta_{\alpha \beta \gamma \delta} \eta^{\mu \nu \zeta \delta}\right) g^{\beta \varepsilon} \Gamma_{\zeta \varepsilon}^{\gamma}=\frac{1}{2} \delta_{\alpha \beta \gamma}^{\mu \nu \zeta} g^{\beta \varepsilon} \Gamma_{\zeta \varepsilon}{ }^{\gamma} .
$$

If we use again the determinant representation (23) of Kronecker, we find

$$
{ }^{\star} \mathcal{F}^{\mu \nu}{ }_{\alpha}=\frac{1}{2}\left|\begin{array}{ccc}
\delta_{\alpha}^{\mu} & \delta_{\alpha}^{\nu} & \delta_{\alpha}^{\zeta} \\
g^{\mu \varepsilon} & g^{\nu \varepsilon} & g^{\zeta \varepsilon} \\
\Gamma_{\zeta \varepsilon}{ }^{\mu} & \Gamma_{\zeta \varepsilon}{ }^{\nu} & \Gamma_{\zeta \varepsilon}{ }^{\zeta}
\end{array}\right| .
$$

This is the generalization of Freud's result to a RiemannCartan spacetime. 


\section{Freud in metric-affine gravity}

Let us finally turn to metric-affine gravity; see [15]. We will denote densities by Gothic letters. The Freud 2-form density and its diamond dual are then defined as

$$
\begin{gathered}
F_{\alpha}:=\frac{1}{2} \mathfrak{g}^{\gamma \delta} \epsilon_{\alpha \beta \gamma} \wedge \Gamma_{\delta}^{\beta}, \\
\mathfrak{A}_{\alpha}:={ }^{\diamond} F_{\alpha}=\frac{1}{2} \mathfrak{g}^{\gamma \delta \diamond}\left(\epsilon_{\alpha \beta \gamma} \wedge \Gamma_{\delta}^{\beta}\right)=\frac{1}{2} \mathfrak{g}^{\gamma \delta} \epsilon_{\alpha \beta \gamma \varepsilon}{ }^{\diamond}\left(\vartheta^{\varepsilon} \wedge \Gamma_{\delta}^{\beta}\right) \\
=\frac{1}{2} \epsilon_{\alpha \beta \gamma \varepsilon} \mathfrak{g}^{\gamma \delta} \Gamma_{\zeta \delta} \beta \diamond\left(\vartheta^{\varepsilon} \wedge \vartheta^{\zeta}\right)=\frac{1}{2} \epsilon_{\alpha \beta \gamma \varepsilon} \mathfrak{g}^{\gamma \delta} \Gamma_{\zeta \delta} \underbrace{\epsilon^{\varepsilon \zeta}}_{2 \text {-form }}
\end{gathered}
$$

Let us now determine the components of the 2-form $\mathfrak{A}_{\alpha}$. For the 2-form $\epsilon^{\varepsilon \zeta}$, we have $\epsilon^{\varepsilon \zeta}=\frac{1}{2} \epsilon^{\varepsilon \zeta \mu \nu} \vartheta_{\mu} \wedge \vartheta_{\nu}$, with $\vartheta_{\mu}:=g_{\mu \kappa} \vartheta^{\kappa}$. Thus, we have $\mathfrak{A}_{\alpha}=\frac{1}{2} \mathfrak{H}^{\mu \nu}{ }_{\alpha} \vartheta_{\mu} \wedge \vartheta_{\nu}$ or

$$
\mathfrak{A}^{\mu \nu}{ }_{\alpha}=\frac{1}{2} \epsilon_{\alpha \beta \gamma \varepsilon} \epsilon^{\varepsilon \zeta \mu \nu} \mathfrak{g}^{\gamma \delta} \Gamma_{\zeta \delta}^{\beta}=\frac{1}{2} \delta_{\alpha \beta \gamma}^{\mu \zeta \nu} \mathfrak{g}^{\gamma \delta} \Gamma_{\zeta \delta}{ }^{\beta} .
$$

Our final result,

$$
\mathfrak{A}^{\mu \nu}{ }_{\alpha}=\frac{1}{2} \delta_{\alpha \beta \gamma}^{\mu \nu \zeta} \mathfrak{g}^{\beta \delta} \Gamma_{\zeta \delta}{ }^{\gamma}=\frac{1}{2}\left|\begin{array}{ccc}
\delta_{\alpha}^{\mu} & \delta_{\alpha}^{\nu} & \delta_{\alpha}^{\zeta} \\
\mathfrak{g}^{\mu \varepsilon} & \mathfrak{g}^{\nu \varepsilon} & \mathfrak{g}^{\zeta \varepsilon} \\
\Gamma_{\zeta \varepsilon}{ }^{\mu} & \Gamma_{\zeta \varepsilon}{ }^{\nu} & \Gamma_{\zeta \varepsilon}{ }^{\zeta}
\end{array}\right|,
$$

coincides with the corresponding Riemannian result in (22). Observe, however, that here we are in the most general metric-affine space. It seems very likely to us that Freud was aware of this generalization. Recall that he even called his 2-form $\mathfrak{A}$ affine tensor density ("Affintensordichte"). ${ }^{3}$

If we take the connection in a metric-affine space and decompose it, we find Schouten [17]; see also [[15] Sec. 3.10] and [[39] Eq. (2.132)],

\footnotetext{
${ }^{3}$ The historically genuine Freud 2 -form is then really $\mathfrak{A}_{\alpha}$, but, conventionally, it is $\mathcal{F}^{\alpha}$ that is named after Freud; see also Mielke [36-38], for example.
}

$$
\begin{aligned}
\Gamma_{\alpha \beta}= & \left.\left.\frac{1}{2} d g_{\alpha \beta}+\left(e_{[\alpha}\right\rfloor d g_{\beta] \gamma}\right) \vartheta^{\gamma}+e_{[\alpha}\right\rfloor C_{\beta]} \\
& \left.\left.-\frac{1}{2}\left(e_{\alpha}\right\rfloor e_{\beta}\right\rfloor C_{\gamma}\right) \vartheta^{\gamma} \quad(\text { Riemann }) \\
& \left.\left.\left.-e_{[\alpha}\right\rfloor T_{\beta]}+\frac{1}{2}\left(e_{\alpha}\right\rfloor e_{\beta}\right\rfloor T_{\gamma}\right) \vartheta^{\gamma} \quad \text { (Rie-Cartan) } \\
& \left.+\frac{1}{2} Q_{\alpha \beta}+\left(e_{[\alpha}\right\rfloor Q_{\beta] \gamma}\right) \vartheta^{\gamma} \quad \text { (metric-affine) } .
\end{aligned}
$$

Here $C_{\alpha}$ is the object of the anholonomity 2-form and $Q_{\alpha \beta}$ the nonmetricity 1 -form. By substituting (51) into (47) and (48), we can find the expressions $\mathcal{F}_{\alpha}$ and $\mathfrak{A}_{\alpha}$ for the corresponding geometries. Of course, Freud's result is exactly recovered in the case of Riemannian geometry.

\section{CONCLUSIONS AND DISCUSSION}

We revisited some well-known results on the superpotential originally introduced by Freud. By approaching this subject using exterior calculus, the definition of the superpotential becomes natural and straightforward, while the usual tensor calculus approach lacks clarity. In doing so, we were able to extend these results to the most general case of metric-affine theories of gravity which may also contain torsion and nonmetricity.

When rewriting the gravitational field equation using the superpotential, the equations take the form of a Yang-Mills type theory. This statement holds for all metric-affine theories and hence includes general relativity. The only drawback of this formulation is that it is based on pseudotensors rather than tensors. While the complete field equations transform correctly under coordinate transformations, the individual parts do not. A similar issue is encountered when considering the teleparallel formulation of general relativity. There the theory is invariant under local Lorentz transformation; however, many of the individual terms appearing in the theory are not. It would be interesting to apply our result within the teleparallel framework.

\section{ACKNOWLEDGMENTS}

We would like to thank Ecardo Mielke (Mexico City), James Nester (Chungli), and Yuri Obukhov (Moscow) for their most helpful remarks.
[1] C. M. Chen, J. M. Nester, and R. S. Tung, Gravitational energy for GR and Poincaré gauge theories: A covariant Hamiltonian approach, Int. J. Mod. Phys. D 24, 1530026 (2015).
[2] M. Dubois-Violette and J. Madore, Conservation laws and integrability conditions for gravitational and Yang-Mills field equations, Commun. Math. Phys. 108, 213 (1987). 
[3] J. Frauendiener, Geometric description of energymomentum pseudotensors, Classical Quantum Gravity 6, L237 (1989).

[4] Y. Itin, Energy-momentum current for coframe gravity, Classical Quantum Gravity 19, 173 (2002).

[5] Y. Itin, Coframe energy momentum current: Algebraic properties, Gen. Relativ. Gravit. 34, 1819 (2002).

[6] J. M. Nester, A new gravitational energy expression with a simple positivity proof, Phys. Lett. 83A, 241 (1981).

[7] J. M. Nester, General pseudotensors and quasilocal quantities, Classical Quantum Gravity 21, S261 (2004).

[8] L. L. So and J. M. Nester, Gravitational energy-momentum in small regions according to the tetrad-teleparallel expressions, Chin. J. Phys. (Taipei) 47, 10 (2009).

[9] L. B. Szabados, On canonical pseudotensors, Sparling's form and Noether currents, Classical Quantum Gravity 9, 2521 (1992).

[10] W. Thirring and R. Wallner, Lecture Notes in Mathematics (Springer, New York, 1978), Vol. 676, pp. 171-178.

[11] Ph. Freud, On the expressions of total energy and total momentum of a material system in general relativity theory (in German), Ann. Math. 40, 417 (1939).

[12] P. Bamberg and S. Sternberg, A Course in Mathematics for Students of Physics (Cambridge University Press, Cambridge, 1990), Vol. 2.

[13] I. Agricola and T. Friedrich, Global Analysis: Differential Forms in Analysis, Geometry and Physics (American Mathematical Society, Providence, 2001).

[14] F. Scheck, Classical Field Theory, on Electrodynamics, Non-Abelian Gauge Theories and Gravitation (Springer, Heidelberg, 2012).

[15] F. W. Hehl, J. D. McCrea, E. W. Mielke, and Y. Ne'eman, Metric affine gauge theory of gravity: Field equations, Noether identities, world spinors, and breaking of dilation invariance, Phys. Rep. 258, 1 (1995).

[16] F. Gronwald and F. W. Hehl, in International School of Cosmology and Gravitation: 14th Course: Quantum Gravity, Erice, Italy, 1995, edited by P. G. Bergmann et al. (World Scientific, Singapore, 1996), pp. 148-198.

[17] J. A. Schouten, Ricci-Calculus: An Introduction to Tensor Analysis and Its Geometrical Applications (Springer, Berlin, 1954).

[18] J. A. Schouten, Tensor Analysis for Physicists, 2nd ed. (reprinted by Dover, New York, 1989).

[19] I. S. Sokolnikoff, Tensor Analysis (Wiley, New York, 1951).

[20] F. W. Hehl and Yu. N. Obukhov, Foundations of Classical Electrodynamics: Charge, Flux, and Metric (Springer, Birkhäuser, Boston 2003).
[21] A. Einstein, The Meaning of Relativity, 5th ed. (Princeton University Press, Princeton, 1955) [translated from German, first published in 1922].

[22] C. W. Misner, K. S. Thorne, and J. A. Wheeler, Gravitation (Freeman, San Francisco, 1973) pp. 370-371; (republished by Princeton University Press, Princeton, NJ, 2017).

[23] A. Trautman, in Differential Geometry, Symposia Mathematica (Academic Press, London, 1973), Vol. 12, pp. 139-162.

[24] W. Thirring, A Course in Mathematical Physics 2, Classical Field Theory, 2nd ed. (Springer, New York, 1986).

[25] W. Kopczyński, Variational principles for gravity and fluids, Ann. Phys. (N.Y.) 203, 308 (1990).

[26] W. Kopczyński (private communication).

[27] G. A. J. Sparling (unpublished).

[28] E. Schrödinger, Space-Time Structure (reprinted with corrections by Cambridge University Press, Cambridge, England, 1960).

[29] A. Trautman, in Encyclopedia of Mathematical Physics, edited by J.-P. Françoise et al. (Elsevier, Oxford, 2006), Vol. 2, pp. 189-195.

[30] Gauge Theories of Gravitation, a Reader with Commentaries, edited by M. Blagojević and F. W. Hehl (Imperial College Press, London 2013).

[31] Yu. N. Obukhov, Poincaré gauge gravity: Selected topics, Int. J. Geom. Methods Mod. Phys. 03, 95 (2006).

[32] Yu. N. Obukhov, Poincaré gauge gravity: An overview, Int. J. Geom. Meth. Mod. Phys. (to be published).

[33] Yu. N. Obukhov and G. F. Rubilar, Covariance properties and regularization of conserved currents in tetrad gravity, Phys. Rev. D 73, 124017 (2006).

[34] Yu. N. Obukhov, G. F. Rubilar, and J. G. Pereira, Conserved currents in gravitational models with quasi-invariant Lagrangians: Application to teleparallel gravity, Phys. Rev. D 74, 104007 (2006).

[35] K. Shimizu, Proposal for the proper gravitational energymomentum tensor, Mod. Phys. Lett. A 31, 1650151 (2016).

[36] E. W. Mielke, Ashtekar's complex variables in general relativity and its teleparallelism equivalent, Ann. Phys. (N.Y.) 219, 78 (1992).

[37] E. W. Mielke, Affine generalization of the Komar complex of general relativity, Phys. Rev. D 63, 044018 (2001).

[38] E. W. Mielke, Geometrodynamics of Gauge Fields: On the Geometry of Yang-Mills and Gravitational Gauge Theories, 2nd ed. (Springer, Cham, 2017).

[39] C. G. Böhmer, Introduction to General Relativity and Cosmology (World Scientific, Hackensack, 2017). 